\title{
Helium Ion Beam Lithography on Fullerene Molecular Resists for Sub-10 nm Patterning
}

\author{
Xiaoqing Shi ${ }^{\text {a, }}$, Philip Prewett ${ }^{\text {b }}$, Ejaz Huq ${ }^{\text {b }}$, Darren M. Bagnall ${ }^{\text {c }}$, Alex P. G. Robinson ${ }^{\text {d }}$, Stuart A. Boden ${ }^{\text {a }}$ \\ ${ }^{\text {a }}$ Electronics and Computer Science, University of Southampton, Southampton, SO17 1BJ, UK \\ ${ }^{\mathrm{b}}$ Oxford Scientific Consultants Ltd., 67 High Street, Dorchester on Thames, Oxfordshire, OX10 7HN, UK \\ ${ }^{c}$ Faculty of Engineering, The University of New South Wales, Sydney, NSW 2052, Australia \\ ${ }^{\mathrm{d}}$ School of Chemical Engineering, University of Birmingham, Birmingham, B15 2TT, UK
}

Keywords:
Helium ion beam
lithography
Helium ion microscope
Fullerene
Molecular resist
Nanolithography
Next-generation lithography

\section{Introduction}

For the last four decades, the minimum feature size for microelectronic fabrication has continued to decrease, keeping pace with Moore's law. As the semiconductor industry enters the sub-10 nm regime, the realisation of prototype "beyond Moore" and "beyond CMOS" devices relies on the development of higher resolution patterned techniques. Electron beam lithography (EBL), a long developed mainstream approach in the industry and research, enables fabrication of sub-10 nm pitched lines [1]. However, due to a strong proximity effect, caused by backscattered electrons and the subsequent secondary electrons, higher resolution and denser patterning is severely limited. Helium ion beam lithography (HIBL) is an emerging technique that uses a subnanometre focused beam of helium ions generated in the helium ion microscope (HIM) [2] to expose resist. It shows high resolution, high sensitivity and reduced proximity effect compared to EBL. Reported state-of-the-art HIBL results demonstrate breakthroughs into the sub-10 nm regime with performance comparable to the best EBL achievements but with 1-2 orders of magnitude higher sensitivity [1, 3-4]. Previous HIBL investigations have involved the use of wellestablished EBL resists such as PMMA and HSQ [3-4]. The demonstrated lithographic resolution of HIBL is not yet comparable to its sub-nm probe size and it is believed to be limited by the composition of the resist and subsequent development process. Recent developments in novel molecular resists, such as molecular glasses [5-6] and fullerene derivatives [7] have attracted great interest in the EBL community due to their small molecular size and therefore high

* Corresponding author. Tel: +44 2380596658.

Email address: Xiaoqing.Shi@soton.ac.uk (X. Shi) resolution patterning potential. In this study, the use of a fullerene derivative resist in HIBL is investigated with the aim of bringing pattern resolutions closer to the fundamental limit of the HIM sub-nanometre probe size.

(a)
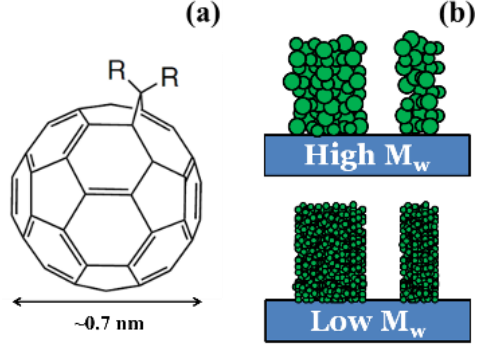

Figure 1 (a) Molecular diagram of generic fullerene resists. R represents the attached side chains to the $\mathrm{C}_{60}$ molecule; (b) Schematic showing the comparison between patterns formed from a high molecular weight resist and those from a low molecular weight resist after development.

The photopolymerisation of fullerene $\mathrm{C}_{60}$ was first demonstrated by Rao et al. and its behaviour as an electron beam resist was described by Tada et al. [8-9]. More recent work has described the development of a new class of negative tone fullerene-based molecular resists [10]. The exposure mechanism for fullerene derivatives is thought to involve fragmentation of the fullerene cage when exposed to the high energy charged particles and the subsequent cross-linking of the remaining material fragments to form an insoluble deposit. With a small molecular size, down to $\sim 0.7 \mathrm{~nm}$, and a molecular weight of $\sim 1000 \mathrm{Mw}$ ( $\mathrm{C}_{60}$ plus the side chains), fullerene resists offer great potential to improve lithographic resolution with a 
reduced line edge roughness (LER) (see Figure 1). Furthermore, non-chemically amplified fullerene derivative resists exhibit high etch durability due to their high carbon content; double that of SAL601 (a high durability commercial resist) and almost eight times than that of silicon in plasma etching [11]. This increased material selectivity is desirable for subsequent pattern transfer in the sub-10 $\mathrm{nm}$ regime. Fullerene derivatives therefore show promise as a feasible resist candidate for next generation lithography (NGL).

The highest resolutions achieved on chemically amplified fullerene resists using electron beam lithography (EBL) are isolated features with a line width of $13.6 \mathrm{~nm}$ as well as $20 \mathrm{~nm}$ lines on a $15 \mathrm{~nm}$ half-pitch [12-13], at a sensitivity of $<50$ $\mu \mathrm{C} / \mathrm{cm}^{2}$. Higher resolutions have been demonstrated in nonchemically amplified fullerene resists. However, these have relatively low sensitivity in EBL (from 200-20,000 $\mu \mathrm{C} / \mathrm{cm}^{2}$; one to three orders of magnitude less than required for commercial resists), which limits their application [10, 14]. In this paper, a study into HIBL with a fullerene-derivative molecular resist is presented, with a focus on resist sensitivity and high resolution, high density patterning.

\section{Experimental}

A methanofullerene derivative of $\mathrm{C}_{60}$ dissolved in anisole (HM01, Irresistible Materials Ltd.) which has not previously been reported as a lithographic material was evaluated. It was examined specifically for the HIBL project on the basis of its size, film formation and solubility properties. The resist was spin-coated onto HF cleaned silicon wafers to a thickness of approximately $10 \mathrm{~nm}$ with a spin speed of $4000 \mathrm{rpm}$. Following a pre-exposure bake on a hotplate at $70{ }^{\circ} \mathrm{C}$ for 5 minutes, the samples were exposed in a HIM (Orion ${ }^{\mathrm{TM}}$ Plus, Carl Zeiss) to a $30 \mathrm{keV}$ focused helium ion beam at a working distance of 7 $\mathrm{mm}$. Helium pressure was maintained at $5 \times 10^{-6}$ Torr during the exposure and a $10 \mu \mathrm{m}$ beam limiting aperture was selected. A beam current of $5 \mathrm{pA}$ was used to expose the large areas efficiently in the resist sensitivity evaluation, using a singlepass, centre to edge writing strategy. For the high resolution patterning, a small beam current of $0.5 \mathrm{pA}$ was applied to achieve optimal beam spot size and the beam followed singlepass lines. The exposed samples were then developed in cyclohexanone for 20 seconds and rinsed in isopropyl alcohol (IPA) for 10 seconds to produce various negative tone patterns, followed by being blow-dried in nitrogen. For a direct comparison of resist sensitivities in HIBL and EBL, the HM01 samples were also exposed in the Gemini FEG-SEM (NVision 40 FIB SEM) to a $30 \mathrm{keV}$ focused electron beam generated from a Schottky source at a working distance of 10 $\mathrm{mm}$. A current of $5.8 \mathrm{nA}$ was used during the exposure with a beam limiting aperture of $120 \mu \mathrm{m}$. The sample preparation and the subsequent development process were identical to that in the HIBL processes. Patterned samples were characterised using both atomic force microscopy (AFM, Multimode ${ }^{\mathrm{TM}} \mathrm{V}$, Veeco) and HIM. The imaging conditions for HIM are a beam energy of $30 \mathrm{keV}$, beam current of $0.5 \mathrm{pA}$ with a working distance of $7 \mathrm{~mm}$. An intermittent-contact (tapping) mode in
AFM with a scanning frequency of $0.723 \mathrm{~Hz}$ was used to obtain the surface depth information.

\section{Results and Discussion}

\subsection{Evaluation of Resist Sensitivity}

An array of $5 \mu \mathrm{m} \times 10 \mu \mathrm{m}$ areas was exposed in HIM with doses ranging from 0 to $288 \mu \mathrm{C} / \mathrm{cm}^{2}$. The residual film thickness after development was measured using AFM. Figure 2 (a) shows an optical micrograph and (b) shows an AFM image of five of the exposed areas. The first dose area appears shallower with a lower contrast compared to the other four. The line profile obtained from the AFM (Figure 2 (c)) confirms a thinner resist remaining for the first area due to underexposure, whilst thicker resist layers remain for other areas that were fully exposed. The dose response curve for HM-01 was determined (see Figure 2 (d)) by plotting the normalized resist thickness against the logarithm of dose, showing that HM-01 exhibits negative tone behaviour with a sensitivity $(50 \%$ of final full exposed thickness) of $\sim 40 \mu \mathrm{C} / \mathrm{cm}^{2}$. An identical procedure was followed on the HM-01 sample exposed in EBL at $30 \mathrm{keV}$ but with a dose range of $0-205,000 \mu \mathrm{C} / \mathrm{cm}^{2}$. The dose response curve is plotted in Figure 2 (d) for comparison. In EBL, the sensitivity of the HM-01 fullerene derivative resist is $20000 \mu \mathrm{C} / \mathrm{cm}^{2}$. Hence, there is a 500 times improvement in sensitivity in $\mathrm{HIBL}$
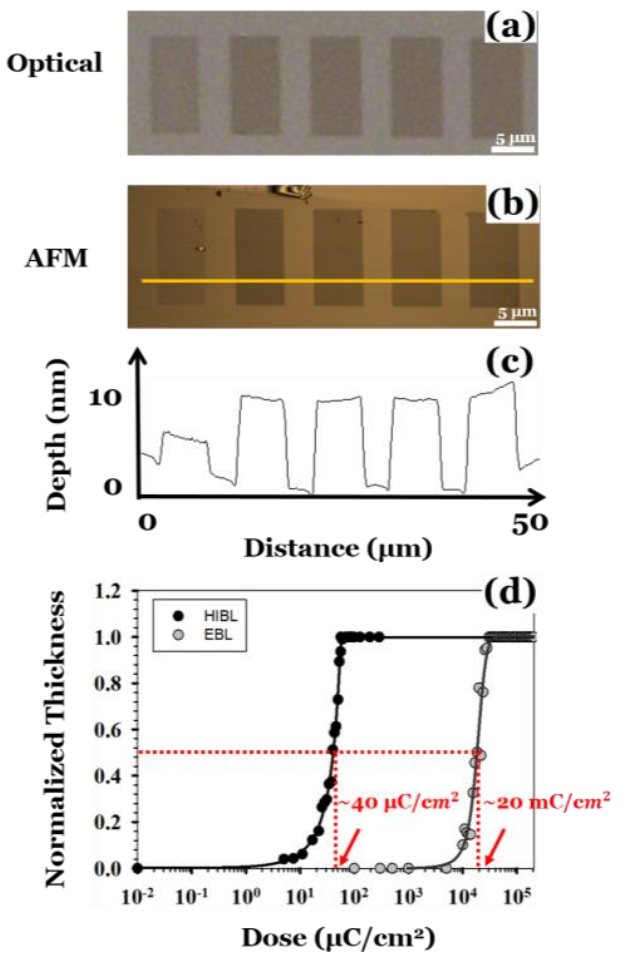

Figure 2. (a) Optical micrograph, (b) AFM image and (c) Line profile (values plotted were averaged from a 20-pixel range) of the same dose test areas on the $\sim 10 \mathrm{~nm}$ fullerene derivative resist (HM-01) patterned using HIBL after development. The applied doses are 37.9, 56.5, 85.0, 128.0 and $192.0 \mu \mathrm{C} / \mathrm{cm}^{2}$ from left to right; (d) Comparison of the dose response curves for HM-01 in HIBL and EBL. The sensitivities are measured to be $40 \mu \mathrm{C} / \mathrm{cm}^{2}$ and $20 \mathrm{mC} / \mathrm{cm}^{2}$, respectively, revealing a 500 times sensitivity improvement in HIBL. 
for this resist, which results in a significantly reduced required current and exposure time, and an improved efficiency. Furthermore, the areal dose used in HIBL can be converted to an ion density of $2.5 \times 10^{14}$ ions $/ \mathrm{cm}^{2}$, which is considered sufficiently low to limit physical damage by ion implantation to the underlying substrate in these regions [15]. This means that HIBL patterning would cause little sub-surface modification, such as bubble formation and swelling, which can limit the highest achievable resolution and prevent subsequent pattern transfer.

\subsection{High Resolution Sparse Feature Patterning}

To find the minimum linewidth (critical dimension) achievable, arrays of $1.5 \mu \mathrm{m}$ long, single-pixel sparsely-spaced lines were exposed with line doses ranging from 0.005 to $0.1 \mathrm{nC} / \mathrm{cm}$. Figure 3 shows HIM images of $40 \mathrm{~nm}$ pitched sparse lines on $\sim 10 \mathrm{~nm}$ thick HM-01 after the development process. The lines are well defined, with high contrast between the patterned resist and the substrate. The linewidths were measured at various locations by the SE contrast from the HIM images with 20-pixel line averaging and the averaged value taken as the critical dimension. The critical dimensions for the single pixel lines vary from 7.3 to $8.3 \mathrm{~nm}$ with different doses. There is discontinuity in line features exposed to 0.045 and $0.06 \mathrm{nC} / \mathrm{cm}$ suggesting under-exposure has occurred. Measurements further reveal that some parts of the lines in Figure 3 (b) are significantly wider, at around $9 \mathrm{~nm}$, which is close to the thickness of the resist layer. This suggests that very thin lines were fabricated by the low dose, however some of these lines collapsed onto the substrate during the subsequent development process due to the high aspect ratio. This results in a greater value of critical dimension in the underexposed pattern compared to that exposed by an optimal dose. The lines with a dose of $0.09 \mathrm{nC} / \mathrm{cm}$ appear thicker due to over-exposure. The optimal line dose was identified to be $0.075 \mathrm{nC} / \mathrm{cm}$, where the smallest critical dimension was achieved.

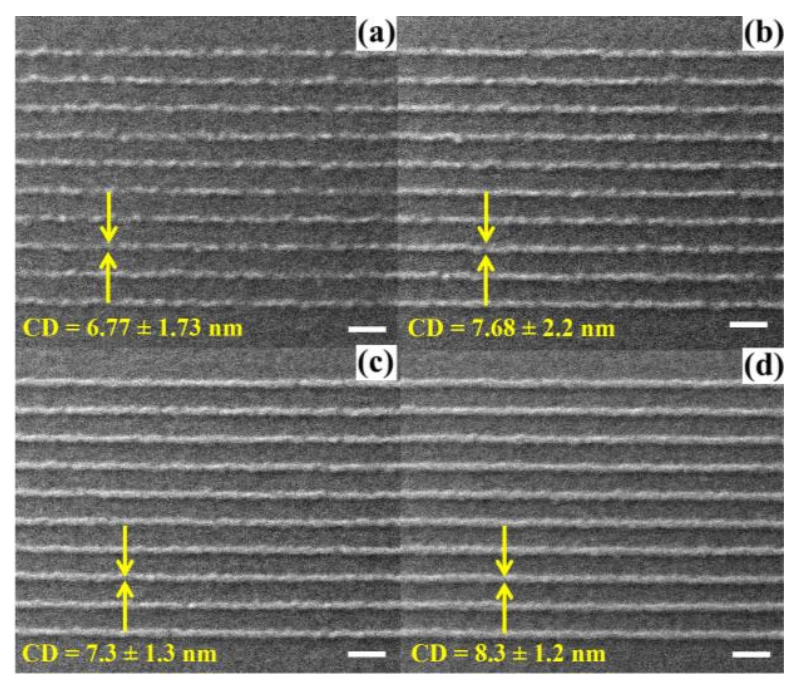

Figure 3. HIM images showing the single pixel sparsely-spaced line patterns exposed on $\sim 10 \mathrm{~nm}$ thick HM-01 resist (line doses are (a) 0.045 , (b) 0.06 , (c) 0.075 and (d) $0.09 \mathrm{nC} / \mathrm{cm}$ ). Scale bar: $50 \mathrm{~nm}$.

\begin{tabular}{|c|c|c|c|c|}
\hline $\begin{array}{c}\text { Line Dose } \\
(\mathrm{nC} / \mathrm{cm})\end{array}$ & 0.045 & 0.06 & 0.075 & 0.09 \\
\hline $\mathrm{CD}(\mathrm{nm})$ & $6.77 \pm 1.73$ & $7.68 \pm 2.2$ & $7.3 \pm 1.3$ & $8.3 \pm 1.2$ \\
\hline LER (nm) & $4.82 \pm 0.22$ & $3.66 \pm 0.12$ & $2.95 \pm 0.06$ & $2.65 \pm 0.08$ \\
\hline
\end{tabular}

Table 1. Critical dimensions (CD) measured by the SE contrast and the line edge roughness (LER) values extracted for line doses of 0.045, 0.06, 0.075 and $0.09 \mathrm{nC} / \mathrm{cm}$ in Figure 3.

To understand the relationship between the line dose and the line edge roughness (LER) in the fabricated line features, the SuMMIT software package (EUV Technology Corp.) was used to extract LER values as shown in Table 1. The results are heavily dependent on the imaging processing methods, hence are not absolute values, but they provide an indication of trend within this study. The greater values of LER in the underexposed patterns were due to the discontinuity in lines. This effect weakens as the dose level rises, hence producing lower LER values. Overall, results show a decrease in resolution when the dose is not optimum, LER decreases when the dose is increased and a low LER was achieved with the optimal dose.

\subsection{High Resolution Dense Feature Patterning}

To determine the highest pattern density of HIBL on HM-01, arrays of densely-packed single-pixel lines with pitches ranging from $30 \mathrm{~nm}$ down to $10 \mathrm{~nm}$ were exposed over a range of doses from 0.005 to $0.1 \mathrm{nC} / \mathrm{cm}$. Examples of HIM secondary electron images of the resulting patterns are presented in Figure 4. Figure 4 (a)-(c) show the effect of decreasing the pitch at a fixed line dose of $0.075 \mathrm{nC} / \mathrm{cm}$. The three pitches are 30, 22 and 14.5 $\mathrm{nm}$ respectively. The results show that the linewidths of all three pitches remain consistent to that of the sparse line features which is $\sim 7.3 \mathrm{~nm}$, indicating a very small proximity effect in HIBL. Figure 4 (d)-(e) show the effect of increasing the line dose at a fixed pitch of $14.5 \mathrm{~nm}$; the three doses are 0.045, 0.075 and $0.09 \mathrm{nC} / \mathrm{cm}$. The measured critical dimensions are $\sim 6.8$, $\sim 7.3$ and $\sim 8.3 \mathrm{~nm}$ respectively, which match to those in the sparse line features (Figure 3 ). The correlation of the dose level and the resulting linewidths is also consistent between the dense and sparse features which has further demonstrated the small proximity effect in HIBL patterning at high density. The best results to date are the continuous and well-defined lines with a pitch of $17 \mathrm{~nm}$ and a 0.5 mark-space ratio (line width of $\sim 8.5 \mathrm{~nm}$ ) as shown in the HIM image and corresponding contrast line profile in Figure 5. This is comparable to the best achievable with state-of-the-art EBL, despite being carried out using a tool not optimized for lithography with standard processing conditions. Figure 6 shows resolvable but broken densely-packed lines with a pitch of $12 \mathrm{~nm}$ and a 0.5 line-space ratio (line width of $\sim 6 \mathrm{~nm}$ ). The discrete charges of the helium ion beam current lead to shot noise which exhibits as a spatial random fluctuation of the helium ion dose received by the resist. Shot noise of the incident beam is considered to affect the critical dimension uniformity and line edge roughness and is a fundamental limit to lithography. In Figure 6, the applied 


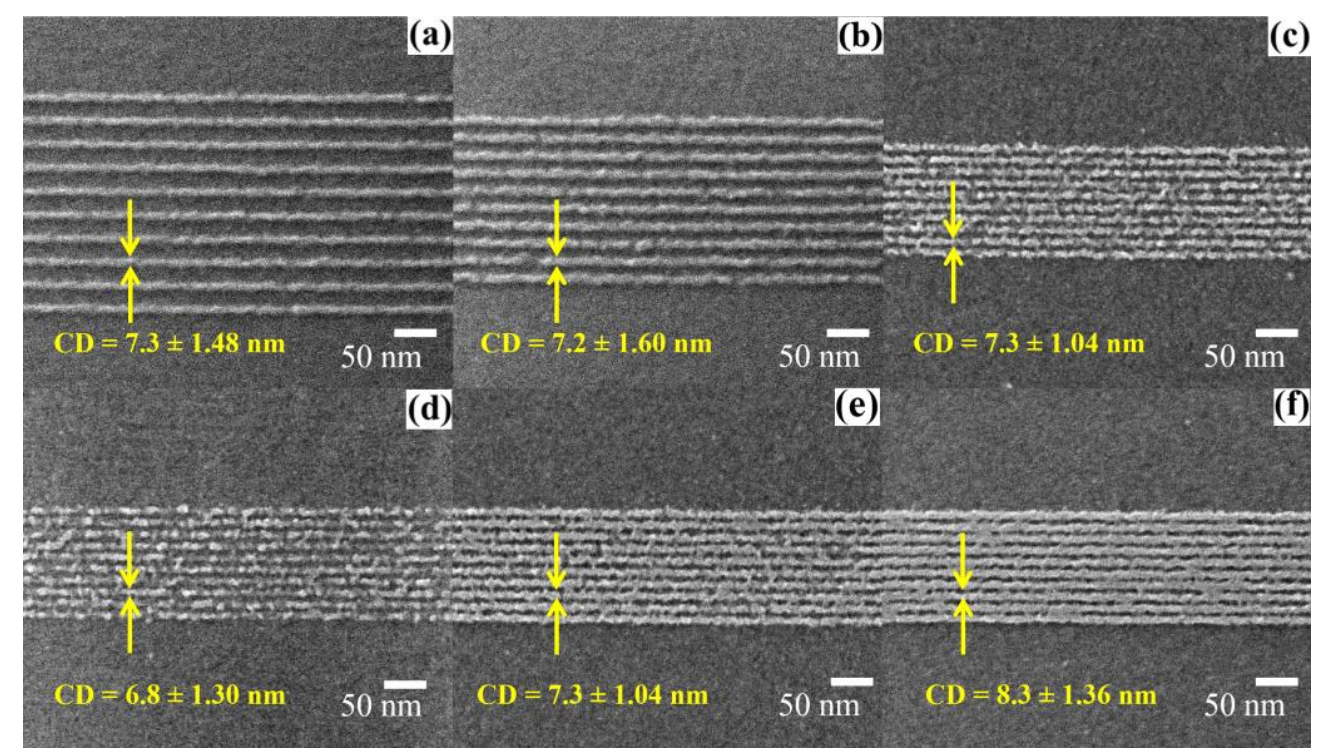

Figure 4. (a)-(c) HIM images show the effect of decreasing the pitch at a fixed line dose of $0.075 \mathrm{nC} / \mathrm{cm}$ (pitches are (a) 30 , (b) 22 and (c) $14.5 \mathrm{~nm}$ ); (d) - (f) HIM images show the effect of increasing the line dose at a fixed $14.5 \mathrm{~nm}$ pitch (line doses are (d) 0.045 , (e) 0.075 and (f) $0.09 \mathrm{nC} / \mathrm{cm})$.

line dose of $0.04 \mathrm{nC} / \mathrm{cm}$ is equivalent to 25 ions/nm, giving a signal-to-noise ratio of 5:1. The subsequent shot noise manifests itself in line discontinuity caused by shot noise dependent missing pixels. The way in which line dose determines this effect is clear in Figure 4 (d)- (f) showing fewer missing pixels and more well-defined lines with increasing dose. This is entirely due to the decrease in shot noise with increasing dose. It can be mitigated by adjusting the scan strategy to reduce pixel to pixel spacing or by partial overlap of adjacent pixels. As the features in Figure 6 approach an aspect ratio of 2:1 it is likely that pattern collapse on development is occurring, hence for pitches below $18 \mathrm{~nm}$ a thinner resist film should be explored. Further improvements could include using a smoother silicon substrate, applying point-of-use resist nanofiltration (with less than $5 \mathrm{~nm}$ pore sizes), varying post exposure bake parameters and developer/development recipe optimisation, with the aim of further improving fidelity of features in the sub $10 \mathrm{~nm}$ regime.
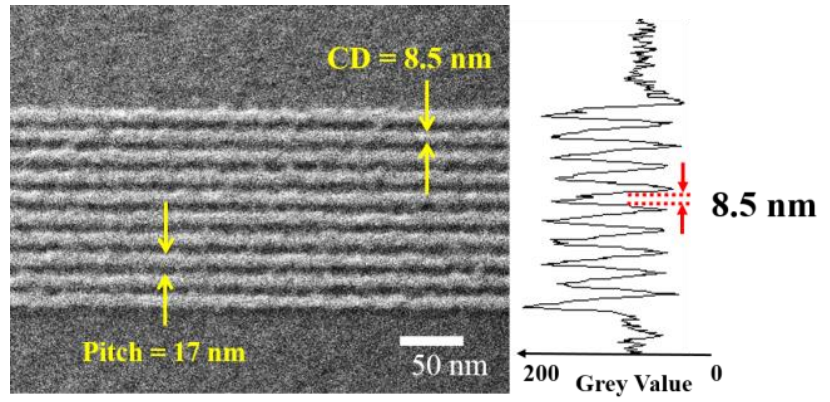

Figure 5. Left: HIM image of dense single pixel features exposed at $0.09 \mathrm{nC} / \mathrm{cm}$ on $\sim 10 \mathrm{~nm}$ thick HM-01 resist. Right: $\mathrm{SE}$ contrast measurement reveals that $17 \mathrm{~nm}$ pitch and $8.5 \mathrm{~nm}$ line width were achieved in the well-defined, continuous lines.

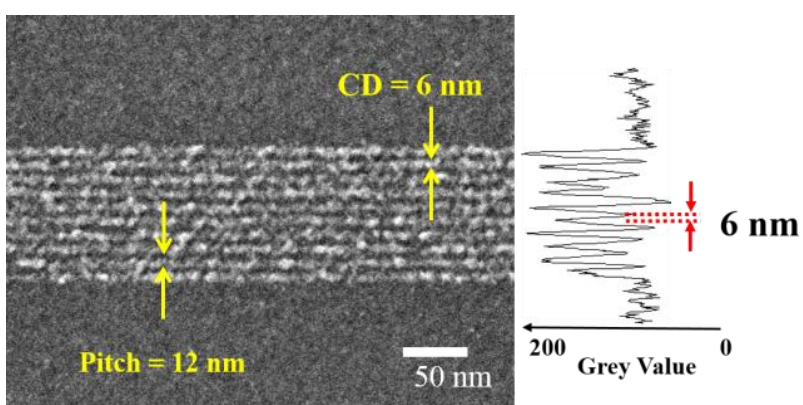

Figure 6. Left: HIM image of dense single pixel features exposed at $0.04 \mathrm{nC} / \mathrm{cm}$ on $\sim 10 \mathrm{~nm}$ HM-01 resist. Right: SE contrast measurement reveals that $12 \mathrm{~nm}$ pitch and $6 \mathrm{~nm}$ line width were achieved in the resolvable but broken lines.

\section{Conclusion}

In summary, a negative tone behaviour with a sensitivity of $\sim 40$ $\mu \mathrm{C} / \mathrm{cm}^{2}$, almost three orders of magnitude more sensitive than that in EBL, was demonstrated in the molecular fullerene derivative resist HM-01 using helium ion beam lithography. To the best of our knowledge, we have demonstrated the best resolution data in a fullerene derivative molecular resist to date using HIBL with standard processing conditions, achieving sub-10 nm patterning with high density and low proximity effect. Further process optimization and pattern transfer development are currently underway with the aim of providing a full assessment of the capabilities and limitations of HIBL in combination with molecular resists as a promising rapid prototyping technique for "beyond Moore" and "beyond CMOS" devices. 


\section{Acknowledgements}

The research leading to these results has received funding from the European Union's Seventh Framework Programme FP7/2007-2013 under grant agreement No. 318804 (SNM).

\section{References}

[1] J. K. W. Yang, B. Cord, H. Duan, K. K. Berggren, J. Klingfus, S.-W. Nam, K.-B. Kim, and M. J. Rooks, "Understanding of hydrogen silsesquioxane electron resist for sub-5-nm-half-pitch lithography," J. Vac. Sci. Technol. B Microelectron. Nanom. Struct., vol. 27, no. 6, p. 2622, 2009.

[2] J. Notte, B. Ward, N. Economou, R. Hill, R. Percival, L. Farkas, and S. McVey, "An introduction to the helium ion microscope," AIP Conf. Proc., vol. 931, pp. 489-496, 2007.

[3] V. Sidorkin, E. van Veldhoven, E. van der Drift, P. Alkemade, H. Salemink, and D. Maas, "Sub-10-nm nanolithography with a scanning helium beam," $J$. Vac. Sci. Technol. B Microelectron. Nanom. Struct., vol. 27, p. L18, 2009.

[4] D. Winston, B. M. Cord, B. Ming, D. C. Bell, W. F. DiNatale, L. A. Stern, A. E. Vladar, M. T. Postek, M. K. Mondol, J. K. W. Yang, and K. K. Berggren, "Scanning-helium-ion-beam lithography with hydrogen silsesquioxane resist," J. Vac. Sci. Technol. B Microelectron. Nanom. Struct., vol. 27, no. 6, p. 2702, 2009.

[5] M. Yoshiiwa, H. Kageyama, Y. Shirota, F. Wakaya, K. Gamo, and M. Takai, "Novel class of low molecular-weight organic resists for nanometer lithography," Appl. Phys. Lett., vol. 69, no. 17, p. 2605, 1996.

[6] T. Kadota, H. Kageyama, F. Wakaya, K. Gamo, and Y. Shirota, "Novel Electron-Beam Molecular Resists with High Resolution and High Sensitivity for Nanometer Lithography," Chem. Lett., vol. 33, no. 6, pp. 706-707, 2004.

H. M. Zaid, A. P. G. Robinson, R. E. Palmer, M. Manickam, and J. A. Preece, "Chemical Amplification of a Triphenylene Molecular Electron Beam Resist," Adv. Funct. Mater., vol. 17, no. 14, pp. 2522-2527, 2007.

[8] A. Rao, P. Zhou, K. Wang, and G. Hager, "Photoinduced polymerization of solid C60 films," Science (80)., vol. 259, no. February, pp. 955-957, 1993.
T. Tada and T. Kanayama, "Nanolithography Using Fullerene Films as an Electron Beam Resist.," $J$. Photopolym. Sci. Technol., vol. 10, no. 4, pp. 647650, 1997.

[10] X. Chen, R. E. Palmer, and A. P. G. Robinson, "A high resolution water soluble fullerene molecular resist for electron beam lithography.," Nanotechnology, vol. 19, no. 27 , p. 275308,2008

[11] J. Manyam, M. Manickam, J. A. Preece, R. E. Palmer, and A. P. G. Robinson, "Plasma Etching of Highresolution Features in a Fullerene Molecular Resist," vol. 7972, no. 0, p. 79722N-79722N-12, 2011.

[12] D. X. Yang, A. Frommhold, X. Xue, R. E. Palmer, and A. P. G. Robinson, "Chemically amplified phenolic fullerene electron beam resist," J. Mater. Chem. C, vol. 2, no. 8, p. 1505, 2014

[13] J. Manyam, F. P. Gibbons, S. Diegoli, M. Manickam, J. A. Preece, R. E. Palmer, and A. P. G. Robinson, "Chemically amplified fullerene resists for e-beam lithography," Proc. SPIE, vol. 6923, no. 0, p. 69230M-69230M-8, 2008.

[14] A. P. G. Robinson, R. E. Palmer, T. Tada, T. Kanayama, E. J. Shelley, and J. A. Preece, "Fullerene Derivatives as Novel Resist Materials for Fabrication of MEMS Devices by Electron Beam Lithography," Proc. Mater. Res. Soc., vol. 546, pp. 219-224, 1999.

[15] R. Livengood, S. Tan, Y. Greenzweig, J. Notte, and S. McVey, "Subsurface damage from helium ions as a function of dose, beam energy, and dose rate," J. Vac. Sci. Technol. B Microelectron. Nanom. Struct., vol. 27, no. 6, p. 3244, 2009. 